CuPAUAM. 20, 1993, pp. 171-183

\title{
DE SITU SIARENSIUM FORTUNALIUM: CORRECCIÓN A PLINIO, N.H. III, 13-14 (BAETURLA CELTICORUM)
}

\author{
Alicia M. CANTO \\ Universidad Autónoma de Madrid
}

Resumé

La célèbre tabula Siarensis n'appartient pas, comment on a dit, au municipium nommé dans Pline, NHIII, 3, 13-14 comme Siarum Fortunalium. Cet oppidum doit être cherché dans la rive droite du flumen Baetis, dans la Baeturia Celticorum plinienne, alors assez loin de "La Cañada". L'auteur propose une nouvelle correction de lecture pour ce discuté et apparemment contradictoire texte de Pline. II faut faire đonc une nouvelle vindicatio pliniana. Cependant, la tabula peut être encore connue comme Siarensis, lors qu'il y a un autre oppidum, stipendiarium, dans la rive gauche du Baetis, du même nom mais sans épithère $(N H I I I, 3,11)$. Ça pose des nouvelles doutes quant à la raison de faire la copie d'un senatus consultum pour une telle, pas privilégiée, ville.

\section{Resumen}

La célebre tabula Siarensis no procede del municipium mencionado en Plinio, NHIII, 3, 13-14 como Siarum Fortunalium. Este oppidum debe ser buscado en la zona derecha del río Guadalquivir, en la Baeturia Celticorum pliniana, y por tanto bastante lejos de "La Cañada". Se ofrece una nueva propuesta de lectura de este controvertido y en apariencia contradictorio texto de Plinio. Se impone, pues, hacer una nueva defensa de la validez de Plinio el Viejo. A pesar de todo, la tabula puede todavía seguir siendo llamada Siarensis, ya que hay otro oppidum del mismo nombre, aunque estipendiario y sin epítetos, en la zona izquierda del Guadalquivir ( $N H I I I, 3,11)$. Ello plantea nuevas dudas sobre el motivo de copiar un senadoconsulto para una ciudad no privilegiada.

\section{SIARUM Y SLARUM FORTUNALIUM}

La celebérrima tabula Siarensis no contiene indicación alguna sobre la ciudad de la que procede. Debe su nombre a la atribución hecha por su primer editor, J. González ${ }^{1}$, basada en su lugar de hallazgo, al

1 J. González y F. Fernández, "Tabula Siarensis", Ium 32, 1981, 1 ss. (primera noticia); J. González, "Tabula Siarensis, Fortunales Siarenses et municipia civiùm Romanorum". ZPE S5, 1984, 55-100 (publicación extensa). Una edición posterior, con correcciones, publicó el mismo autor en Bronces Juridicos romanos de Andalucia, Sevilla 1990, 153 ss. Cf. asimismo las actas del coloquio internacional Estudios sobre la Tabula Siarensis, Scvilla-Utrera 1986 (Anejos de Archivo Español de Anqueologia IX) Madrid 1988 y, más recientemente, el coloquio La commemorazione di Germanico nelle testimonianze epigrafiche: Tabula Hebana e Tabula Siarensis (Casino 1991), en prensa. 
SO. de Utrera (Sevilla) que coincide con la cita de Plinio $N H 3,3,11$ : Oppida Hispalensis conventus... et a laeva Hispal colonia cognomine Romulensis, ex adverso oppidum Osset quod cognominantur Iulia Constantia, $<$ Luc>u/e/rgentum quod Iuli Genius, Orippo, Caura, Siarum, fluvius Maenuba Baeti et ipse a dextro latere infusus. De este texto pueden extraerse dos conclusiones iniciales: 1.a) Un Siarum se encontraba en la margen izquierda del Guadalquivir ${ }^{2}$, frente a Osset y el fluvius Maenuba, y 2.a) No se indica de tal Siarum ningún epíteto, por lo que hay que deducir que es, en época de Plinio, un simple oppidum stipendiarium.

En el primer coloquio de los citados en nota 1 (publ. 1988), Patrick Le Roux fue el encargado de comentar, entre otras cosas, la localización antigua, nombre y estatuto de la ciudad ${ }^{3}$. Él mismo cita el párrafo de Plinio III, 1, 13-14, donde es mencionada por Plinio una comunidad, la de los Siarenses Fortunales, en un texto que fija a esta última, claramente, entre las ciudades de la Beturia céltica, y por tanto, en el lado derecho del Guadalquivir, al otro lado y lejos de la Siarum que acabamos de ver ( $c f$. infra). De este texto, donde Siarum lleva un epíteto, Fortuna, que se suele relacionar, sin mucha base, con Julio César ${ }^{4}$, González y Le Roux dedujeron que se trataba de la misma ciudad, dando primero por sentado un nuevo "error" pliniano.

Creo que ello no es así, puesto que Plinio habla claramente de dos Siarum distintas: una sin epiteto, al S. del Guadalquivir, y otra con epíteto, al N. del Guadalquivir, más próxima al río Ana y fuera, por tanto, de la zona anterior.

Otros dos argumentos se manejan para tal identificación: El primero, la epigrafía de los yacimientos de Zarracatín y La Cañada, ambos en el término de Utrera y separados entre sí unos $3 \mathrm{Km}$. Entre las 21 inscripciones aparecidas con certeza en ambos yacimientos romanos, y recogidas por González en $1986^{5}$, hay cuatro menciones del ordo Siarensium ${ }^{6}$; pero en ninguna de ellas consta el epíteto de Fortunales, que supuestamente debía de ostentar. El segundo, el pedestal CIL II 1371, de Moguerejo (Sevilla), donde nada menos que cinco comunidades (Italicenses, Hispalenses, Asidonenses, Fortunales Siarenses y Aeneanici Callenses) dedican un homenaje fúnebre a Lucia Avircia Aciliana. Sin embargo, creo que de este pedestal no se puede inferir la localización, en la inmediata proximidad, de los Callenses Aeneanici y los Siarenses Fortunales, porque tampoco se ubican alli mismo Hispalis, Asido ni Italica.

Así las cosas, puede decirse que la identificación entre la o las ciudades donde apareciera la tabula Siarensis, y los Siarenses Fortunales de Plinio III, 3, 13-14, no reposa sobre bases ciertas.

Por otra parte, incluso P. Le Roux ya puso de relieve los problemas de atribuir a esta ciudad, en época augustea, la condición de colonia civium Romanorum, y de aceptar que después lo habría cambiado

2 Esta es mi lectura del texto: et a laeva... inicia una relación en la que cstán Hispalis, Lucurgentum, Orippo, Cauray Siarum, con un inciso: ex adverso oppidum Osset.... precisamente para indicar que Osset está en el lado derecho, por donde también afluye al Baetis el rio Maenoba, lo que parce reforıar la expresión et ipse a deatro latere infusus.

3 P. Le Roux, "Siarum et la tabula Siarensis. Statut politique et honneurs religicux en Bétique sous Tibère", op.cit. 21 ss. (donde, pese a considerar aceptable mi opinión sobre la lectura de 3, 1, 11, finalmente opta por unificar ambas referencias de Siarum).

4 El propio Le Roux (op.cit. 24): "( )n pourrait envisager un déplacement des habitants de Siarum sur la rive droite, peutêtre menacts par les migrations [?] ou débordements du Ciuadalquivir, vers des terres plus propices et plus riches? La présence de la Fortuna dans la dénomination suggèrerait des vocux et l'intervention de César ou de ses successeurs dans cette affaire..." Para otra interpretación de este cpíteto, $f f$. mi trahajo en prensa "I a Beturia Céltica: Introducción a su epigrafia", en Célticos y Túndulos: la Beturia (Actas Jornadas del Musco Nacional de Arte Romano. Mérida, marzo 1994), Monografias Emeritenses 10, en prensa.

5 J. González, "Epigrafia del yacimicnto de la Cañada". Actas, op.cis. en n. 1, 91 ss., apartado A.

6 Gonźlez, art.cit., núms. 3.11, 13 y 14: municipium nostrum Siarense, ordo splendidissimus municipi Siarensis o Siarensium son las expresiones utilizadas en los epigrafes donde se pueden restituir estos términos con seguridad. 
por el de municipium iuris Latini, en época de Vespasiano, "al fusionarse dos núcleos antiguos", como defiende González para adecuar al sitio la discutida frase de la tabula (frag b, col. b, lín. 23-27), que trata sobre las ciudades donde debe exponerse públicamente el senadoconsulto. Tal degradación estatutaria es impensable, y en ello ha convenido la crítica posterior ${ }^{7}$.

En el marco de estudios más amplios ${ }^{8}$, dedicados a la época romana de una zona arqueológica e históricamente muy desconocida de la actual región de Extremadura, que corresponde a la antigua Baeturia Celticorum de la antes citada descripción pliniana, 3, 13-14, he catalogado las 218 inscripciones del área (incluyendo la Baeturia actualmente portuguesa) y he intentado, entre otros objetivos, definir su territorio, sus fuentes de riqueza y su caracterización histórica en varios sentidos. Entre ellos, encontrar la razón por la que Julio César, en el reducido espacio de poco más de $600 \mathrm{Km}^{2}$, concedió a cinco de estas ciudades su propio nomen, Iulia, junto con su tribu habitual, la Galeria?.

Naturalmente, ello me ha llevado a tratar de ubicar, con la mayor fiabilidad posible, las siete ciudades mencionadas por el polígrafo, de las que, hasta ahora, lo estaba, con total certeza, sólo una, Nertobriga Concordia Iulia, y con bastantes posibilidades otra, Seria Fama Iulia, en los municipios actuales de Fregenal de la Sierra y Jerez de los Caballeros, respectivamente, ambas en el Suroeste de la actual provincia de Badajoz. Entre las no bien ubicadas, pues, está la célebre Siarum Fortunalium, que se viene llevando al S. del Baetis.

\section{VINDICATIO PLINIANA}

Los párrafos en que Plinio el Viejo nos da noticia, por otra parte bastante detallada, del área en cuestión, se han solido ver como una contradicción ${ }^{10}$, como un descuido de redacción y precisión ${ }^{11} \mathrm{o}$ como problema derivado de la combinación de fuentes heterogéneas, $\mathrm{y}$ a veces incongruentes ${ }^{12}$. La no

7 Cf. C. Castillo Garcia, "El progreso de la epigrafia romana en Hispania (1983-1987)", Emerita 59.2, 1991, 225 ss., especialmente 236-237, donde cita la bibliografia más importante hasta 1987: en su nota 53 recoge las opiniones favorables a la existencia de municipia c. $R$. en época de Tiberio. Cf. asimismo A. M. Canto, "Un veintenio clave para la epigrafia latina de España", Boletin de la Asociación Española de Amigos de la Anqueologia 30-31, 1991, 247 ss., espec. 263, con la bibliografia posterior hasta 1991.

8 A.M. Canto, "Noticias arqueológicas y epigráficas de la Beturia Céltica", Cuadernos de Prehistoria y Anqueologia de la Universidad Autónoma de Madrid 18, 1991, 275 ss.; cad., "CIL II 997 ("Zafra") y los Sosii Prisci de la Bética", Anas 4-5, 19911992, 13 ss.; ead., art. cit. supra en n. 4: cad., Epigrafia romana de la Beturia Céltica (F. R. B. C.), en prensa; ead., "La Baeturia Celticorum: Problemas y soluciones", en elaboración.

9 Para la tribu Galeria en Hispania, of. R. Wiegels, Die Tribusinschriften des römischen Hispanien. Ein Katalog (Madrider Forschungen 13), Berlin 1985, y C. Castillo, "La tribu Galeria en Hispania: ciudades y ciudadanos", Estudios sobre la Tabula Siarensis, cit. supra en n. 1, 233 ss. Las ciudades en cuestión son Seria Fama Iulia, Nertobriga Concordia Iulia, Segida Restituta Iulia, Ugultunia Contributa Iulia y Lacimurga Constantia Iulia.

${ }^{10}$ E. Hübner, CIL II, p. 182: "...sat gravis oritur dubitatio... " A. Tovar, Iberische Landeskunde, II.1 (Baetica), Baden-Baden, 1974 , p. 31 con n. 35 o p. 153 con n. 101; R. Wiegels, Tribus, passim.

11 Por ejemplo, E. Albertini, Les divisions administratives de l'Espagne romaine, París, 1923, p. 92; L. Garcia Iglesias, "La Beturia, un problema geográfico de la Hispania Antigua", AFA 44, 1971, p. 86-108 (en adelante Garcia Iglesias, Beturia, H. Galsterer, Untersuchungen zum römischen Städtewesen auf der iberischen Halbinsel (MF 8), Berlin 1971, 4; B. D. Hoyos, "Pliny the Elder's titled Bactican Towns: Obscuritics, Frrors and Origins". Historia 28, 1979, p. 439-471 (sin duda el más duro), seguido, entre otros, por A.U. Stylow, MM 28, 1987, 57 ss., espec, 58 con n. 6. Un reciente estado de la cuestión publicó M. Mayer, "Plinio el Viejo y las ciudades de la Baetica. Aproximación a un estado actual del problema", Fstudios sobre Uro, Colonia Iulia Generiva, Sevilla, 1989, p. 303-333), quien habla (p. 316) de "una descriptio voluntariamente alcjada de la realidad contemporánea."

12 R. Corzo y A. Jiménez, "Organización territorial de la Bética", AFA 53, 1980, p. 21-48, espec. 21-22. 
resolución del problema ha contribuído además a mantener recelo y desconfianza hacia la única fuente literaria de que en realidad disponemos, tanto para el estudio de las divisiones administrativas de la Hispania romana, como para la caracterización jurídica y geográfica de sus ciudades.

A la defensa de Plinio he dedicado hace algunos años otras páginas, a propósito del papel fronterizo del Guadiana ${ }^{13}$. Hay que recordar, por orra parte, que el último y más detenido trabajo sobre la Beturia, desde el punto de vista histórico, cuenta ya con más de veinte años desde su publicación, y se debió a L. García Iglesias ${ }^{14}$. Para comodidad del lector, me permito citar a continuación literalmente los párrafos plinianos en cuestión $(3,13-14)^{15}$ :

13 Quae autem regio a Baete ad fuvium Anam tendit extra praedicta, Baeturia appellatur, in duas divisa partes totidemque gentes: Celticos, qui Lusitaniam attingunt, Hispalensis conventus, Turdulos, qui Lusitaniam et Tarraconensem accolunt, iura Cordubam petunt. Celticos a Celtiberis ex Lusitania advenisse manifestum est sacris, lingua, oppidorum vocabulis, quae cognominibus in Baetica distinguntur: 14 Seriae adicitur Fama Iulia, Nertobrigae Concordia lulia, Segidae Restituta Iulia, Contributa Iulia Ugultuniae, cum qua et Curiga nunc est, Lacimurgae Constantia Iulia, Siarensibus ${ }^{16}$ Forrunales et Callensibus Aeneanici. praeter haec in Celtica Acinippo, Arunda, Arucci-Turobriga ${ }^{17}$, Lastigi, Salpesa, Saepone, Serippo. Altera Baeturia, quam diximus Turdulorum et conventus Cordubensis, habet oppida non ignobilia Arsam, Mellariam, Mirobrigam, Reginam, Sosintigi $i^{18}$, Sisaponem ${ }^{19}$.

${ }^{13}$ A. M. Canto, "Colonia Iulia Augusta Emerita. Consideraciones cn torno a su fundación y territorio", Gerión 7, 1989, p. 149-205.

14 L. García Iglesias, Beturia (cit. n. 11). Con anterioridad, pueden citarse los capítulos correspondientes a inscripciones de Zafra y su entorno por R. Caro, Adiciones al Principado y Antigüedad de Sevilla (msc. de 1634), Sevilla, 1932, p. 45 y 47 (que son anexos a los capitulos 66 y $70 \mathrm{del} \mathrm{libro} 3$ de su Antigüedad y Principado de la llustrisima ciudad de Sevilla y Corografia de su convento jurídico, Sevilla 1634), asi como un curioso trabajo debido a M. Pérez Quintero, La Beturia vindicada, Sevilla, 1794. Recientemente, yo misma he hecho ya dos aportaciones a la cuestión, cf. "Noticias arqueológicas y epigráficas de la Beturia Cética", CuPAUAM 18, 1991, p. 275-298 y "La Beturia Céltica: Introducción a su epigrafia", Célticos y Türdulos, Jornadas del Museo Nacional de Arte Romano, Mérida, 1994 (en prensa).

15 C. Plinius Secundus, Naturalis Historia, I, edd. C. Mayhoff y L. lan, Leipzig, 1906 (reimp. 1967), p. 236-237.

16 Todos los msc. dan, incorrectamente, Steresibi/Teresibus, corregido en la citada edición como Steresibus. La aparición del epigrafe de Montellano (Sevilla), estudiado en primer lugar por F. Fita $(B R A H 31,1897$, p. $387=E E \mathrm{VIII}, 306$ ) resolvio aquella extraña lectura, al mencionar a los mismos Fortunales Siarens(es), de donde las ediciones debian ser corregidas.

17 Ya E. Albertini (Divisions, p. 87 con n. 1), para explicar esta excepción en una lista claramente alfaberica, supuso, en mi opinión con acierto, que Turobriga debia estar agrupada con Arucci, basándose en el epigrafe CIL II, 964, cf. n. 34.

18 Una ciudad de nombre Sosontigi puede localizarse en o cerca de Alcaudete, Jaén (cf. por último A. U. Stylow, "Inscripciones latinas del sur de la provincia de Cóndoha", Gerión 1, 1983, p. 267-303, espec. 282 ss., que la cree mal localizada por Plinio, cf. aquí nota final 1, p. 23 con n. 75). Como apuntó ya Garcia lglesias (Beturia, p. 103), la distancia hasta esta zona es excesiva, por lo que no debe tratarse de la misma ciudad. ya que la Sosonsigi jienense está dentro del convento astigitano y la túrdula Sosintigi pertenecia al cordobés (asi ya Tovar, Baetica, 119). No seria la única ciudad de esta serie no bien localizada: Arsa se viene poniendo con muchas dudas en Azuaga, por lo que he preferido seguir la ubicación aproximada según Ptolomeo II, 4, 10 (sureste de Fornacis, Hornachos, BA). Para las demás hay acuerdo en, respectivamente, Fuente Obejuna, Capilla y Reina, y para Sisapo of. la nota siguiente.

19 La tradicional localización en el importantísimo centro minero de Almadén (Ciudad Real) no se comprucba bien por la arqueología. P. Sillières ("Sisapo: Prospections et découvertes", AEA 53, 1980, p. 49-57) propuso el yacimiento ibero-romano del Cerro de las Monas. Alli podria ubicarse, según creo, la Sisapo Nova. La Sisapo Vetus (ambas ciudades túrdulas menciona Estrabón: 3, 2, 3), podría en cambio situarse en otro yacimiento próximo, la Bienvenida, término de Almodóvar del Campo, Ciudad Real (C. Fernández Ochoa y A. Caballero Klink, "Nuevo documento epigráfico para la localización de Sisapo", CuPAUAM 9-10, 1982-1983, p. 211-220), debido a la antigüedad de sus materiales, que remontan al menos al siglo $\mathrm{V}$ a.C. 
Las principal acusación de inexactitud a Plinio en este párrafo se plantea por la frase praeter haec in Celtica Acinippo... puesto que, después de definir la Baeturia en general como "el área comprendida entre los ríos Ana y Baetis" ${ }^{20}$, relaciona una serie de ciudades que están claramente fuera, e incluso muy lejos, de

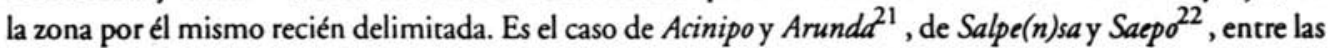
bien documentadas arqueológica o epigráficamente ${ }^{23}$. Esta situación ha llevado a dar como evidente la existencia de un error, o al menos una contradicción, en el trato de la Beturia por Plinio. Mi hipótesis a este respecto es que tal error no existe, y que estamos ante un problema posterior y puramente textual.

\section{DEFINICION DE LAS TRES LISTAS}

Al tratar de la Beturia, Plinio diferencia con la mayor claridad tres grupos de ciudades: El primero es el de la Baeturia Celticorum, el tercero el de la Baeturia Turdulorum, y en medio de estos dos, otro grupo de ciudades, ordenadas alfabéticamente como las túrdulas. Esta serie intermedia va vinculada con la primera, no sólo por el praeter haec que la introduce, sino también porque los topónimos se corresponden, efectivamente, con radicales indoeuropeos ${ }^{24}$. Los dos grupos principales, primero y tercero, se diferencian nítidamente, tal como Plinio los caracteriza. Para mayor claridad comento separadamente cada grupo de rasgos:

1) Carácter común a ambas partes de la Beturia: Están en la tegión que se extiende desde el Betis hacia el río Anas, y ocupadas por otras tantas gentes: Celtici y Turduli.

2) Caracteres específicos de la Beturia de los Célticos: la distingue porque es confinante con Lusitania y pertenece jurídicamente al convento de Hispalis. Cuenta con siete ciudades principales (de las cuales sólo dos carecen del epíteto de Iulia), a las que se añade, (nunc), una más.

3) Caracteres específicos de la Beturia de los Túrdulos: la distingue porque limita tanto con Lusitania como con Tarraconense y depende en lo judicial del convento de Corduba. Incluye seis ciudades, que Plinio define como non ignobilia.

Las ciudades del primer grupo, continúa el polígrafo, es claro que son producto de una antigua inmigración de celtíberos, procedentes en segunda instancia de Lusitania ${ }^{25}$. Ello se veía, según él, en que

20 En lo que viene a coincidir con una frase de Estrabón (3, 2, 3): "Tal es, igualmente, el aspecto (árido) de la Beturia, que tiene seco el territorio que bordea el curso del Anas."

21 Actuales Ronda la Vicja y Ronda, en la scrrania de Málaga. Cf. A. Tovar, Baetica, p. 153 y 154: R. Wiegels, Tribus, p. 11-15.

22 Salpe(n)sa en Facialcázar, cerca de Utrera, Sevilla. Cf. CII. II, 1963 (lex municipii Flavii Salpensani) y García Iglesias, Beturia, p. 100. Saepo (ciudad distinta de Usaepo), cerca de las tres anteriores citadas, en el cortijo de Vistalegre, término de Olvera (Cádiz), cf. infra n. 41.

23 Cf. García Iglesias, Beturia, passim, y aqui, infra, para los comentarios sobre cada una de las ciudades.

24 A. Tovar, Baetica, passim, aunque apunta a los dos sufijos en -ipo (Acinipo. Seripo), como autóctonos de origen mediterráneo, pero mezclados con radicąles célticos.

25 A. Tovar, "Les Celtes en Bétique", Érudes Celriques 10, 1963, p. 365, proponía eliminar del texto pliniano las palabras a Lusizania como superfluas, entendiendo que los topónimos reduplicados se referian directamente a Celtiberia, donde hay, por ejemplo, una Nertobriga y una Segeda. Pero más adelante (p. 366 con n. 1) reconocía que la numerosa onomástica bética similar a la lusitana podía dar la razón al texto de Plinio. Cf. razones de parentesco entre célticos y turdetanos, en Estrabón 3, 2, 15 (siguiendo a Polibio). Otros argumentos, arqueológicos c históricos, pueden también aportarse. 
conservaban aún sus antiguos ritos, su lengua e incluso los topónimos primitivos de sus ciudades, si bien las béticas se distinguían de las lusitanas porque tenían cognomina. Algunos de estos topónimos, efectivamente, se conservan también en la Celtiberia de origen, como los citados Segeda o Nertobriga ${ }^{26}$. Pero, además, y como veremos infra, los siete, o al menos la mayoría, de los nombres de ciudades de la Beturia céltica, debían ser reduplicación de nombres iguales que debemos buscar en la provincia lusitana. Un detalle éste que ha solido pasar desapercibido en la bibliografía sobre la descripción pliniana de Bética y Lusitania ${ }^{27}$. El hecho creo debe tenerse por cierto, ya que, al comenzar su descripción de Lusitania, Plinio vuelve sobre ello: "...stipendiarios... praeter iam dictos in Baeticae cognominibus..." 28 .

De la precisión geográfica, etnográfica y jurídica de Plinio en esta zona, parece que podemos inferir, metodológicamente, que, para afirmar de una ciudad su pertenencia a la Baeturia Celticorum, se deben dar las tres condiciones a la vez: a) no puede estar situada al S. del Guadalquivir; b) debe estar más próxima a Lusitania y c) debe pertenecer al convento hispalense ${ }^{29}$.

Estas premisas son indispensables para la correcta comprensión de la lista intermedia: Las siguientes siete ciudades que cita (con la peculiaridad de que su orden es alfabético) no es lícito entenderlas como betúricas ${ }^{30}$ : Son ciudades que, teniendo un origen también céltico, y conservando seguramente algo de la lengua o de los ritos, incumplen una o más de las tres condiciones prescritas: $\mathrm{O}$ no están en la zona interfluvial en cuestión, o no lindan con Lusitania, o no dependen del convento hispalense. ¿Por qué aparecen, pues, en este punto? Porque el minucioso Plinio ${ }^{31}$ consideró más adecuado incluirlas aquí, en virtud de sus rasgos célticos, que donde administrativa o geográficamente les hubiera correspondido. Por ello procede a mencionarlas separadamente, introduciendo la frase con un praeter haec que, si bien es cierto significa usualmente "además de", también es posible documentar como "más allá de", "fuera de", "aparte de" 32 y, en este caso, daría en mi opinión más rotundidad a la idea de que tales ciudades, precisamente, no formaban parte de la Baeturia Celticorum.

26 A. Tovar, op.cit. en n. 10. II.3, Tarraconensis, Baden-Baden 1989, p. 412 y 414.

27 Sólo he encontrado, en texda la bibliografia. la opinión claramente favorable a esta deducción de Albertini, Divisions, cit. en n. 11, p. 57 con n. 2.

28 Plinio, NH, 4, 118 : "...ciudades (lusitanas), además de las ya dichas por sus cognomina béticos...". Hay que localizar, pues, en Lusitania, tal como dijo Albertini. al menos otras cinco ciudades: Seria, Nertobriga, Segida, Ugultuniay Laci(ni)murga, sin epítetos. De ellas, creo que podemos tener ya referencia de dos. Una es Laci(ni)munga, ciudad vetona en Cogolludo, junto al embalse de Orellana (Canto, Gerión 7, 1989). F.n segundo lugar, la noticia de la toma por M. Marcelo de la ciudad de Nerkobriga, en el 152 a.C., no debe referirse a la celtibérica, como se viene creyendo ( $c f$. A. Tovar, Tarneonensis, p. 414, C-526), sino a la lusitana de igual nombre, aunque su localización la ignoramos. Habria, pues, en Hispania al menos tres ciudades de nombre Nertobriga. Los casos de Siarum, Callet y Curiga, como se verá, pueden ser distintos.

29 Lo mismo cabe decir, por cierto. de las ciudades túrdulas: además de estar entre ambos rios, sus territorios deben "verter" hacia Lusitania y Tarraconense y pertenecer judicialmente al convento cordobés (de ahí que haya de suponerse una Sosontigi distinta de la betúrica en Alcaudete. Jaén. ya que la ciudad del mismo nombre que se situaba alli debió pertenecer al convento astigitano),

30 Asi lo hacen R. Corzo y A. Jiménez. art.cit. supra en nota 12, p. 23 (su lista n. ${ }^{\circ}$ 8), como la mayoría de los autores que han tratado la cuestión.

31 Para detalles sobre su precisión y escrupulosidad (incredibile studium, opus eruditum..., etc.) of. los comentarios de su sobrino Plinio el Joven (Epist. 3. 5). Se olvida a veces que hablamos de un procurador y consejero imperial, que tuvo un destino administrativo en la peninsula, y cuyos libros de comentarios (opisthographos quidem et minutissimis scripros) quiso, sin éxito, comprar el gobernador Larcio Licinio por una alta suma.

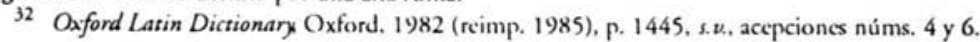


Creo, pues, que esta segunda lista, lejos de expresar contradicción o error, descuido o desorden en la redacción, lo que evidencia es una metodología rigurosa, como era exactamente esperable en el gran enciclopedista y procurador imperial ${ }^{33}$. Naturalmente, para probarlo hay que resolver antes algunas cuestiones. Como el hecho de que todas las ediciones filológicas de Plinio dan la frase praeter haec "in Celtica...", lo que aparenta ser contradictorio con la premisa anterior. De ello hablaré más adelante.

\section{INCUMPLIMIENTO DE LAS CONDICIONES}

De momento, quizá sea de interés ver cómo cada una de estas siete ciudades intermedias incumple una o más de las condiciones:

Acinipo. No está entre ambos ríos; no está cerca de Lusitania; no es del convento hispalense.

Arunda. Caso idéntico al anterior.

Arucci-Turobriga. Debe tratarse aquí de una ciudad con dos nombres o, mejor, con dos asentamientos; no deben ser dos ciudades diferentes, como se suele $\mathrm{creer}^{34}$, porque se rompería el orden alfabético, como bien decía Albertini y, por otra parte, los dos nombres aparecen bien asociados por medio de la diosa Ataecina ${ }^{35}$. La existencia epigráfica de la segunda se ha confirmado recientemente ${ }^{36}$. Me parece, pues, una suerte de contributio entre un núcleo urbano y una ciudad-santuario. Bastante bien localizada en la zona de Aroche (Huelva) ${ }^{37}$, está, por lo tanto, entre el Guadiana y el Guadalquivir. La proximidad a Lusitania es estrecha. No se encuentra, sin embargo, "en las áridas tierras a lo largo del curso del Anas" sino en una altura superior a los $700 \mathrm{~m}$.; ni en la cuenca del río Ardila, sino en la del Chanza. Visto todo lo cual, la razón de su exclusión por Plinio puede ser que no dependiera del convento hispalense, sino del gaditano, a pesar de la dificultad que sugerir tal hipótesis conlleva ${ }^{38}, \mathrm{o}$, simplemente, que pertenecía a otra cuenca fluvial, fuera de la Beturia.

33 Plinio el Joven, loc.cit. en n. 31. habla sobre la amicitia principum de la que gozaba su tío, especialmente con Vespasiano, con el cual despachaba desde antes del alba y del que recibió officia maxima. Todos estos condicionantes debian ser más tenidos en cuenta cuando se analizan sus libros geográficos.

34 Cf. García Iglesias, Beturia, p. 97-98, y A. Tovar, Baetica, p. 171-173. García Iglesias refiere toda la problemática sobre las dos Arucci, Nova y Vetus. El mismo autor (p. 99 con n. 48) proponia la zona de Corte de Messangil, cercana a Moura (Port.), es decir, Arucci Nova según CIL II 963, para el asentamiento de Turobriga y su correspondiente culto a Ataecina. Pero Arucci Nova nunca existió ( $c f$. HEp. 3, 1993, 197) y, aunque Corte de Messangil es un importante santuario, el principal de Ataecina debia estar en San Mamés, cerca de Aroche, cf. nota siguiente. Como expondré en otro lugar, es fácil que el topónimo real, o el más antiguo, fuera Turibriga, con i.

35 El epigrafe CIL II, 964, de una sacerdotisa turobrigense y aparecido en Aroche (Huelva), topónimo que ha conservado perfectamente el Arucciantiguo. Las muestras del culto de esta diosa-madre de la ultratumba continúan aumentando. Uno de sus santuarios principales debió estar en la iglesia de Sta. Lucia del Trampal, en Alcuéscar (Cáceres), de la que se han publicado recientemente diez títulos: HEp. 2, 1990, 192-201. Cuatro que conservan el topónimo dicen Turibrigensis, con i.

${ }^{36}$ Cf. J. Esteban Ortega, "Epigrafe sobre la diosa Ataecina en Bienvenida (Badajoz)", Actas de las II Jornadas de Metodologia y Didactica de la Historia (Historia Antigua. Cäceres 1981), Cáceres 1984, p. 21-25, interesante porque menciona Dominal Atraegina: $\beta$ Turobrigae, es decir la diosa "de Turóbriga", sin adjetivar el topónimo, Ataecina Turobrigensis, como es más habitual. Por cierto que ello justamente descartaria en principio la ubicación de la ciudad alli. En este sentido, cf. un turobrigensis en Aroche (HEp. 3, 1993, n. ${ }^{203}$ ) y una fistula con posible lectura M(unicipii) T(urobrigensis) f(istula aquaria), cf. HEp. 3, 1993, 205).

37 J. González, Corpus de Inscripciones Latinas de Andalucia (CILA) I. Huelva, Sevilla, 1989, p. 24: Opina que son dos ciudades, y que Arucci podría situarse en el yacimiento de Fuente Seca, mientras Turobriga quizá en el área de la Ermita de San Mames.

38 De estos aspectos trato en un trabajo en preparación sobre la Hispania pliniana. 
Lastigi. Si aceptamos que la única ciudad arestiguada de este nombre es la citada por Plinio en 3 , 12, como también creía García Iglesias, se admitirá que correspondía al convento astigitano; tampoco estaba entre los ríos ni próxima a Lusitania ${ }^{39}$.

Salpe(n)sa. Al S. del Betis, cerca de Utrera; era del convento hispalense, pero incumplía las otras dos condiciones.

Saepo. Su situación parece ahora clara en torno al Cortijo de Vistalegre, término de Olvera $(C a ́ d i z)^{40}$. Es, por lo tanto, distinta de la \{U\}saepo mencionada por Plinio en 3, 15, en último lugar entre las del convento gaditano ${ }^{41}$. Esta Saepo no se encontraba entre el Guadiana y el Guadalquivir, ni cercana a Lusitania.

Serippo. Uno de los topónimos que más gráficamente representa la mixtura indigeno-celtismo. Se ha propuesto su localización en Los Molares, al SE. de Utrera ${ }^{42}$. Que se trate, como se ha propuesto, de la Irippo atestiguada por monedas ${ }^{43}$, o de la gaditana Belippoy la citada Irippo a la vez ${ }^{44}$, debe ser descartado, ya que, en una lista alfabética y tras una ciudad comenzada con la letra $S$, no pueden esperarse ni Belippo ni Irippo. Tampoco se menciona el topónimo en los itinerarios. Sigue siendo, pues, de localización incierta, si bien el hecho mismo de aparecer en esta lista, en la que no sería una excepción, nos permite suponer que incumplía uno o más de los citados condicionantes.

Como conclusión, ninguna de las ciudades de la lista intermedia podría ser incluída por Plinio dentro de la zona por él mismo caracterizada como Baeturia Celticorum. No puede, pues, considerárselas betúricas.

\section{PRAETER HAEC IN $<B A E>T I C A$}

Pero estas siete ciudades, sin embargo, aparte de su estirpe céltica, sí comparten con las anteriores algo más: Son béticas. Mi propuesta, por tanto, para solventar esta aparente y secular contradicción, es aceptar una

39 D. Detlefsen, "Die Geographie der Provinz Bätica bei Plinius (NH III, 6-17)", Philologus, 30, 1870, p. 265 ss., espec. 278 y E. Hübner, en CIL II, p. 122. Recuérdese además que la corrección del final de esta frase: Olontigi, Laelia, Lastigi, se debe a Th. Mommsen (CIL II, p. 194), donde los msc. traian alontigicaeli, olontigicaeli et alastigi, alostigi, etc. ( $f$. aparato critico de la edición y la discusión en el art.cit. de Detlefsen). Existe la posibilidad de dos Lastigi, una en el convento astigitano y otra en el hispalense, pero para ello hay que valorar la presencia de monedas de Lastigi en las cercanias del río Guadiamar, lo que hoy no parece admitirse. En cualquier caso, y contra lo que se cree, las dobles menciones de la misma ciudad en Plinio son rarísimas: Cuando repite nombres es porque se refiere a ciudades diferentes.

40 Cf. HEp. 2, 1990, n.॰ 267 (Olvera, Cádiz). Esta inscripción confirma el aserto pliniano sobre dos ciudades distintas de nombre Saepa. La primera, conocida de antiguo, en la Dehesa de la Fantasía, cerca de Cortes de la Frontera (Málaga), en la tribu Galeria, más meridional y en el convento gaditano, de la que siempre se creyó hablaba Plinio ( $c f$., por ejemplo, García Iglesias, Beturia, 100). La segunda, la ahora conocida, clevada por los flavios, de tribu Quirina, unos $40 \mathrm{Km}$. al N. de la anterior y en el convento hispalense. Plinio debe referirse como de estirpe céltica a esta última.

${ }^{41} \mathrm{Ya}_{\mathrm{a}}$ Wiegels, Tribus, p. 65, s.u (U?)saepo), dudaba de la Vinicial. Frente a Detlefsen, Albertini o Galsterer, tenían razón Hübner (CIL II, p. 180), Garcia Iglesias y Tovar. Fsta V es la inicial del cpíteto Victrí; como ya se veía, más explayado y según Hübner, en la lin. 9 de CIL II, 1341.

42 A. Tovar, Baetica, p. 152, citando la opinión de Céán Bermúdez pero dando a la ciudad como "sonst unbekannte".

43 ld., Baetica, p. 152: E. Hübner, Monumenta l.inguae Ibericae, Berlin, 1893, n. 171; Antonio VrVES, La moneda hispdinica, Madrid, 1924-1926, 3, p. 93.

44 Asi R. Corzo y A. Jiménč, art.cit., p. 27 con n. 17. 
modificación en la transmisión manuscrita del texto pliniano, leyendo al principio de esta lista praeter haec in $<$ Bae >tica Acinippo... Con ello voy en realidad en la misma dirección que K. Müller, en su comentario a la edición de Ptolomeo ${ }^{45}$, aunque por otra vía, puesto que Müller lo que propuso, por cierto que sin mucho éxito, fue la supresión de la preposición in: praeter haec fin\} celtica Acinippo... Ello es sólo ligeramente más difícil de aceptar desde el punto de vista de la tradición textual. Pero el sentido es parecido. El sentido sería, para Müller: "Además de estas (ciudades) (son) célticas... Acinipo...etc." ${ }^{46}$. Con mi propuesta se entendería: "Aparte de estas (ciudades)(son también de origen céltico) en la Bética (estas otras): Acinipo..." Es decir, para concretarlo en el estilo pliniano: Praeter haec (oppida celtica origine) in Baetica Acinipo... etc."

La tradición manuscrita, recogida por lan-Mayhoff, no registra ninguna variante para la palabra celtica. Los filólogos admiten con dificultad este tipo de correcciones en los manuscritos, pero hay ocasiones en que la lógica o los criterios arqueológicos pueden acabar imponiéndose. Hay un argumento suplementario: La frase, como se viene entendiendo, es irregular en Plinio, al entender in (Baeturia) celtica o, lo que se cree igual, in Celtica, pues Plinio habla de Baeturia Celticorum. Que la expresión sería rara en él se prueba algo más adelante cuando, de acuerdo con el precedente, deberíamos esperar: altera Baeturia, quam diximus Turdula... y, sin embargo, dice quam diximus Turdulorum... Ésta, la referencia al étnico en genitivo plural, es una costumbre en Plinio, que se puede documentar en otros pasos de la misma descripción de Hispania ${ }^{47}$. Por lo tanto, si la expresión completa "Baeturia Celtica" no existió en el uso de Plinio, menos se puede aceptar su elisión, in Celtica, como se ha venido haciendo con la frase que comento. Además, la Celtica, sustantivada, es la palabra propia para referirse a Galia-Germania ${ }^{48}$.

El error manuscrito es bien explicable: La terminación, -tica, es igual para ambas palabras, y sólo restaría admitir una confusión paleográfica de Bae- por Cel-. Para un copista desinformado sería incluso lógica aquí la palabra celtica, ya que se venía hablando de los Celtici. Y no es ni mucho menos el primer caso en el que la tradición manuscrita pliniana se presenta en desacuerdo con topónimos que la evidencia epigráfica posterior ha demostrado erróneos.

Sin ir más lejos, éste que propongo sería el quinto fallo de los manuscritos sólo en este párrafo 3 , 14: el AruncilArungi textual se ha confirmado Arucci, tanto a través de Ptolomeo como epigráficamente ${ }^{49}$. Regiones osintigi/osintiadi-s apareció como corrupto ante Regina/Sosintigi ${ }^{50}$. El aparente error en las versiones Lacimurga/Lacinimurga lo he tratado en otro lugar ${ }^{51}$. Una tercera vacilación notable es la que afecta a la mención de Ugultunia ${ }^{52}$. El cuarto caso constatado es el de los Steresibi/Teresibi Fortunales.

45 K. Müller y C. Fischer, Claudii Ptolomaei Geogmphika, Paris, 1883-1900 (comentarios).

46 Es decir: Praeter haec (sunt oppida) Celtica...

47 Por cjemplo, en 3, 18: ...conventus Asturum... conventus Bracarum..., en 3, 20: ... regio Ilergaonum..., en 3, 21: regio Ilergetum..., en 4, 110-111: saltus Vasconum... Vardulorum oppida... regio Cantabrorum... o, fuera de Hispania, en 3, 38: ... regio Volcarum Tectosagum..., entre otros muchos que se podrian aducir.

48 En el sentido de "pais de los celtas", asi en Plinio. NH4, 105 (Galia) o 6, 34 (Europa central), y Vitrubio 8, 2, 6 (Europa central, la zona del Rhin, distinta de Galia).

49 Cf. supra, notas 34 a 36. Ptolomeo 2, 4, 11, cita a Aroukki entre los "béticos célticos".

50 L. García Iglesias, Beturia, p. 102-103. I a corrección Regina, Sosintigi, remonta a Detlefsen, p. 304, y ha sido generalmente aceptada, a pesar de las dudas de E. Hübner (CIL II, p. 837). Como ya he apuntado, debe haber dos Sosintigi, la túrdula en lugar aún desconocido.

51 A. M. Canto, art.cit. supra en n. 13,186 y ss.

52 L García Iglesias, Beturia, 91. La vacilación manuscrita está entre Ugultuniacum qua et Curiga nunc est y Ugultunia, cum qua et Curiga nunc est... Cf. A.T. Fear, "Contributa Iulia, Ugulsunia and Curiga", Gerión 9, 1991, $151-161$. 
fue el P. Fita (y no A. Schulten ${ }^{53}$ ), quien propuso ya su corrección en Siarenses, Siarensibus, cuando publicó en $1897^{54}$ el epígrafe de El Arahal (Sevilla), al que ya me he referido. El nombre procedía, efectivamente, del topónimo Siarum/Searo ${ }^{55}$. La confusión Ste-por Sia-no se aleja mucho o no es mucho más difícil de concebir que la de Cel-por Bae-y, en todo caso, la existencia de otros cuatro errores en el mismo párrafo en todos los manuscritos creo que da cierta base para admitir un quinto.

Parece oportuno recordar ahora, en apoyo de esta tesis, que Ptolomeo $(2,4,11)$ relaciona a Aroukki, Aroúnda y Akinippó (junto a Koúrgia y Ouáma) como ciudades "de los béticos célticos". Aunque los casi ochenta ańos de romanidad transcurridos entre Plinio y Ptolomeo hacen no directamente comparables sus informaciones, es posible que Ptolomeo guarde aquí el recuerdo de una "Bética céltica". Cabe decir también que, para mediados del siglo II d.C., los betúricos son para él ya sólo "turdetanos" $(2,4,10)$, es decir, que se ha perdido la diferenciación entre célticos y túrdulos que había en la Beturia hasta época de Plinio (o la precisión en los datos).

Si se acepta mi hipótesis quedaría resuelto el "problema" de la Beturia céltica. Plinio no tuvo un error de concepción, ni confusiones en cuanto a la delimitación exacta de esta zona. Sí existió el "fenómeno de asociación", que García Iglesias sugirió en $1971^{56}$, pero no fue inconsciente: Tras citar las ciudades que formaban parte, geográficamente, de la Beturia de los Célticos, y antes de enumerar las de los Túrdulos, le pareció oportuno relacionar otra serie de ciudades béticas cuyo origen céltico era en su época aún patente, sacándolas de su orden administrativo (conventual y geográfico) y ordenándolas alfabéticamente, puesto que todas eran estipendiarias. Para hacer más clara la propuesta, presento un sucinto mapa con la localización conocida o supuesta de las ciudades de los tres grupos (fig. 1).

\section{COROLARIO PARA LA TABULA SIARENSIS}

Mediante esta propuesta de corrección en la tradición pliniana manuscrita del párrafo concreto 3, 13-14, no sólo se reivindica al autor, sino que se desprende una segunda conclusión, que afecta al origen de uno de los más célebres bronces de estos años. Si se terminan las especulaciones a partir de pretendidas "inexactitudes" plinianas, no es tan fácil llevar ciudades descritas como de la Baeturia Celticorum hasta el S. del Guadalquivir, tal como se ha hecho con una Siarum no privilegiada.

Todas las ciudades célticas, pues, de la primera lista pliniana debieron estar entre el Baetis y el Ana y ninguna al Sur del Baetis. De esta constatación se deduce que, en principio, Siarum Fortunalium, como Callet Aeneanicorum, deben ser buscadas al Norte del río Baetis (y muy lejos, por tanto, de los yacimientos de La Cañada y Zarracarín, de donde procede nuestra tabula Siarensis). Para el problema concreto de la tabula Siarensis no es indispensable saber ahora dónde estuvo exactamente la ciudad. Pero una buena candidata, en mi opinión, es Montemolín, al E. de Llerena y NO. de Monesterio (Badajoz). Junto a otras razones que no es lugar aquí para mencionar, de allí procede una inscripción funeraria (CIL II 6337), cuyas tres primeras líneas

53 Como creía A. Tovar. Bética, 146 con n. 96.

\$4 F. Fita. "Los Callenses Acneanici del Arahal y de Montellano". BRAH31, 1897, 381 ss.: Se trata de un epigrafe fúnebre honorifico, costeado por varias ciudades béticas.

55 Searo es nombre de ciudad que acuña moneda autóctona, cf. A. Vives, La moneda hispdinica, Madrid 1926, 3, p. 84 ss.

56 Beturia, 108. 
rezan: L(ucio) Nor[bano - f(ilio) Gal(eria tribu?)/ Mensori IIvir(o) [q(uin)q(ennali)?]/ bis IIvir(o) [aed(ili)? - - J. Aun siendo funeraria, no es mal indicio de la proximidad de un núcleo urbano la existencia de un duovir, que además estuvo inscrito en la tribu Galeria, y por ello no es atribuíble a la ciudad de Emerita Augusta, contiguos a cuya praefectura Mullicensis ${ }^{57}$ creo vivieron los verdaderos Siarenses Fortunales.

\section{CONCLUSIONES}

Así pues, la ciudad de los Siarenses Fortunales se localizaría en el suroeste de la actual provincia de Badajoz y de la antigua Baeturia Celticorum, en las cercanías de los pueblos de Montemolín y/o Valencia del Ventoso, y limitando su territorio con los de la praefectura Mullicensis de la colonia de Mérida, a modo de exclave lusitano dentro de la provincia de Bérica.

La segunda conclusión es que hay que aceptar la existencia de dos Siarum y tres Callet, en un fenómeno tan habitual en la Hispania antigua como es el de la reduplicación de topónimos; y, naturalmente, reconocer una vez más que mucho de lo que no entendemos de C. Plinio Secundo se debe a nuestra falta de conocimientos y no a su precisión.

La tercera es que los yacimientos de La Cañada y Zarracatín (Sevilla), lugar de aparición de muchos e importantes epígrafes, no acogieron ninguna ciudad privilegiada o con epítetos, sino un simple oppidum stipendiarium. En los alrededores, y especialmente al sur de Utrera, la patria de Rodrigo Caro, se acumulan yacimientos importantes, que debieron contar con tierras muy ricas y grandes latifundios. Por allí han aparecido también los documentos de Salpensa y, en muy distintos sitios, distantes entre 5 y $15 \mathrm{Km}$. de Utrera, muchas inscripciones de familias tan influyentes como los Messii Rustici, como J. González y A. Caballos se han encargado de destacar ${ }^{58}$.

Las varias inscripciones que mencionan al splendidissimus ordo Siarensium, a la res publica Siarensis, lo hacen siempre a una Siarum sin epíteros (¿y por qué no hacerlo, si los hubiera tenido, y fueran cesarianos?). Como mucho, la ciudad sería colectivamente un municipio de derecho latino menor antes de Vespasiano, o lo recibiría de Domiciano; quizá los Messii, los Acilii, los Aemilii o los Avircii, fueran en origen sólo los ricos, las primeras familias romanizadas, de ciudadanía cesariana o augustea, (tribu Galeria, pero viritim) de las élites municipales, pero cuyo ascenso al Senado se produce, quizá ya muy contadamente, en época flavia, y se mantiene y acrecienta con los emperadores béticos, como homines novi del senado flavio y trajano-adrianeo.

Desde este nuevo punto de vista, si en La Cañada sólo había un oppidum stipendiarium, no puede mantenerse, pues, como ya había extrañado a la crítica, que hubiera aquí ninguna colonia civium Romanorum, pero ahora vemos que ni siquiera un municipium civium Romanorum.

¿Por qué aparece aquí una copia del senadoconsulto para honrar la memoria de Germánico? Esta pregunta debe quedar, de momento, sin respuesta. Por lo menos, y por ahora, gracias a este humilde Siarum reencontrado en Plinio III, 3, 11, podemos seguir denominándola tabula Siarensis...

57 A.M. Canto, art. cit. supra en nota 13.

58 J. González, art.cit. coloquio, 91-126, los cataloga todos; A. Caballos, Los senadores hispanorromanos y la romanización de Hispania (siglos I-III), t. I, Prosopografia (I-II), Fcija 1990. 


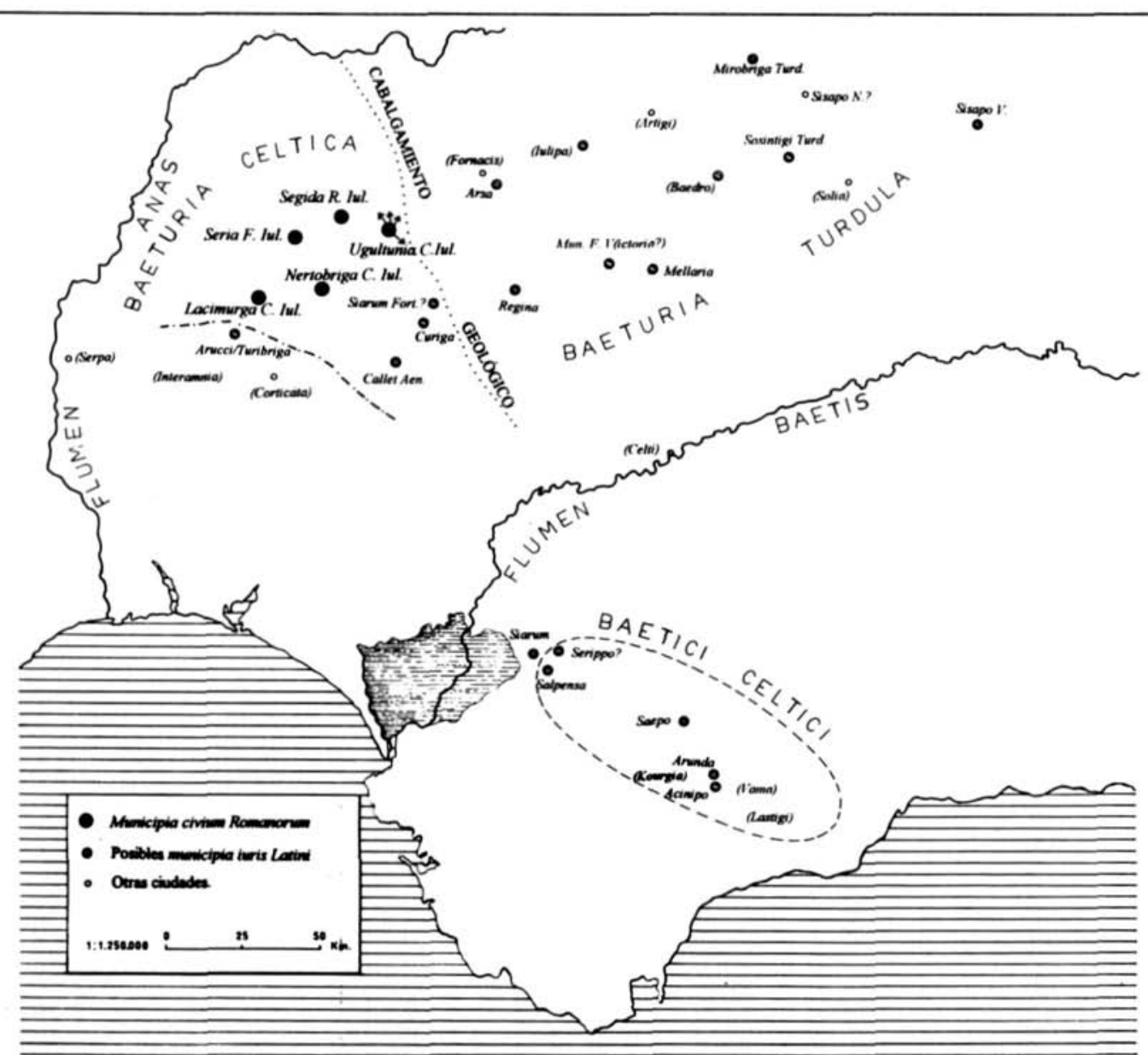

Figura 1. Mapa de propuesta de distribución de las ciudades de la Beturia ceftica y túrdula, a ambos lados del cabalgamiento geológico de Ossa Morena. Ciudades de origen celtico en otros puntos de la Bética, según Plinio el Viejo y Ptolomeo (Mapa de A. Canto y S. Luzón) 
Localizaciones modernas del mapa de la fig. 1 (los puntos no son exactos, se refieren a yacimientos confirmados o que sugiero en los términos municipales señalados).

\section{BAETURIA CELTICORUM:}

Seria Fama Iulia: Jerez de los Caballeros (BA)

Nertobriga Concordia Iulia. Fregenal de la Sierra (BA)

Segida Restituta Iulia. Burguillos del Cerro? (BA)

Ugultunia Contributa Iulia: Los Santos de Maimona-Zafra-Alconera-Medina de las Torres-Fuente de Cantos (BA)

Curiga. Monesterio (BA)

Lacimurga Constantia Iulia: Encinasola? (H)

Siarum Fortunalium: Montemolín? (BA)

Callet Aeneanicorum: Cala-Minas de Cala? (H)

Serpa. Serpa (Port.)

Corticata. Cortegana (H.)

Interamnia. en la zona, sin hipótesis de localización.

\section{BAETURIA TURDULORUM:}

Arsa: sureste de Fornacis-Hornachos (BA)

Mellaria. Fuente Obejuna (CO)

Mirobriga. Capilla (BA)

Regina: Casas de Reina (BA)

Sosintigi: zona de Santa Eufemia (CO)

Sisapo Vetus: Almodóvar del Campo (CR)

Sisapo Nova. Almadén? (CR)

Iulipa. Zalamea de la Serena (BA)

Artigi: cerca de Castuera? (BA)

Baedro: Hinojosa del Duque/Belalcázar (CO)

Mun. F V(ictoria?): Azuaga (BA)

Solia: El Guijo (CO) $)^{59}$

\section{BAETICI CELTICI:}

Arucci/Turibriga. Aroche/San Mamés (H)

Acinippo. Ronda la Vieja (MA)

Arunda. Ronda (MA)

Lastigi: zona al SO. de Ronda, sin hipótesis de localización.

Salpensa: Facialcázar (SE)

Saepo: Olvera (CA)

Serippo: en la zona, sin hipótesis de localización.

Kourgia: al N. de Acinippo, según Ptolomeo.

Vama. al O. de Acinippo, según Ptolomeo.

Celtri: Peñaflor (SE).

59. Para las propuestas de localización de Artigi, Baedro y Solia, la atribución del Municipium Flavium V(ictoria.), y el comentario de otros núcleos epigráficos túrdulos, of. A. U. Stylow, "El Municipium Flavium V(-) de Azuaga (Badajoz) y la municipalización de la Baeturia Turdulorum", Studia Historica. Historia Antigua IX, 1991, 11-27. 